\title{
Monotonicity rule for the quotient of two functions and its application
}

\author{
Zhen-Hang Yang ${ }^{1}$, Wei-Mao Qian², Yu-Ming Chu ${ }^{1 *}$ and Wen Zhang ${ }^{3}$
}

\section{"Correspondence:}

chuyuming2005@126.com

'Department of Mathematics,

Huzhou University, Huzhou, 313000,

China

Full list of author information is

available at the end of the article

\begin{abstract}
In the article, we provide a monotonicity rule for the function $[P(x)+A(x)] /[P(x)+B(x)]$, where $P(x)$ is a positive differentiable and decreasing function defined on $(-R, R)$ $(R>0)$, and $A(x)=\sum_{n=n_{0}}^{\infty} a_{n} x^{n}$ and $B(x)=\sum_{n=n_{0}}^{\infty} b_{n} x^{n}$ are two real power series converging on $(-R, R)$ such that the sequence $\left\{a_{n} / b_{n}\right\}_{n=n_{0}}^{\infty}$ is increasing (decreasing) with $a_{n_{0}} / b_{n_{0}} \geq(\leq) 1$ and $b_{n}>0$ for all $n \geq n_{0}$. As applications, we present new bounds for the complete elliptic integral $\mathcal{E}(r)=\int_{0}^{\pi / 2} \sqrt{1-r^{2} \sin ^{2} t} d t(0<r<1)$ of the second kind.
\end{abstract}

MSC: $26 \mathrm{~A} 48 ; 33 \mathrm{E} 05$

Keywords: monotonicity rule; complete elliptic integral; absolute error; relative error

\section{Introduction}

The most commonly used monotonicity rule in elementary calculus is that $f$ is increasing (decreasing) on $[a, b]$ if $f:[a, b] \rightarrow \mathbb{R}$ is continuous on $[a, b]$ and has a positive (negative) derivative on $(a, b)$, and it can be proved easily by the Lagrange mean value theorem. The functions whose monotonicity we prove in this way are usually polynomials, rational functions, or other elementary functions. But we often find that the derivative of a quotient of two functions is quite messy and the process is tedious. Therefore, the improvements, generalizations and refinements of the method for proving monotonicity of quotients have attracted the attention of many researchers.

In 1955, Biernacki and Krzyż [1] (see also [2], Lemma 2.1, [3]) found an important criterion for the monotonicity of the quotient of power series as follows.

Theorem 1.1 ([1]) Let $A(t)=\sum_{k=0}^{\infty} a_{k} t^{k}$ and $B(t)=\sum_{k=0}^{\infty} b_{k} t^{k}$ be two real power series converging on $(-r, r)(r>0)$ with $b_{k}>0$ for all $k$. If the non-constant sequence $\left\{a_{k} / b_{k}\right\}$ is increasing (decreasing) for all $k$, then the function $t \mapsto A(t) / B(t)$ is strictly increasing (decreasing) on $(0, r)$.

In [4], Cheeger et al. presented the monotonicity rule for the quotient of two functions.

Theorem 1.2 ([4]) If $f$ and $g$ are positive integrable functions on $\mathbb{R}$ such that $f / g$ is decreasing, then the function $x \mapsto \int_{0}^{x} f(t) d t / \int_{0}^{x} g(t) d t$ is decreasing.

(c) The Author(s) 2017. This article is distributed under the terms of the Creative Commons Attribution 4.0 International License (http://creativecommons.org/licenses/by/4.0/), which permits unrestricted use, distribution, and reproduction in any medium, provided you give appropriate credit to the original author(s) and the source, provide a link to the Creative Commons license, and indicate if changes were made. 
Unaware of Theorem 1.2, Anderson et al. [5], Lemma 2.2 (see also [6], Theorem 1.25) established l'Hôpital's monotone rule that can be applied to a wide class of quotients of functions.

Theorem $1.3([5])$ Let $-\infty<a<b<\infty, f, g:[a, b] \rightarrow \mathbb{R}$ be continuous on $[a, b]$ and differentiable on $(a, b)$, and $g^{\prime}(x) \neq 0$ on $(a, b)$. If $f^{\prime}(x) / g^{\prime}(x)$ is increasing (decreasing) on $(a, b)$, then so are the functions

$$
\frac{f(x)-f(a)}{g(x)-g(a)}, \quad \frac{f(x)-f(b)}{g(x)-g(b)} .
$$

If $f^{\prime}(x) / g^{\prime}(x)$ is strictly monotone, then the monotonicity in the conclusion is also strict.

Pinelis [7] provided the following monotonicity theorem.

Theorem 1.4 ([7]) Let $f$ and $g$ be differentiable and $g^{\prime}$ never vanish on an open interval $(a, b) \subset \mathbb{R}$. Then the following statements are true:

(1) If $g g^{\prime}>0$ on $(a, b)$, limsup $\operatorname{su}_{x \downarrow a} g^{2}(x)(f(x) / g(x))^{\prime} /\left|g^{\prime}(x)\right| \geq 0$ and $f^{\prime} / g^{\prime}$ is increasing on $(a, b)$, then $(f / g)^{\prime}>0$ on $(a, b)$.

(2) If $g g^{\prime}>0$ on $(a, b), \liminf _{x \downarrow a} g^{2}(x)(f(x) / g(x))^{\prime} /\left|g^{\prime}(x)\right| \leq 0$ and $f^{\prime} / g^{\prime}$ is decreasing on $(a, b)$, then $(f / g)^{\prime}<0$ on $(a, b)$.

(3) If $g g^{\prime}<0$ on $(a, b), \liminf _{x \uparrow b} g^{2}(x)(f(x) / g(x))^{\prime} /\left|g^{\prime}(x)\right| \leq 0$ and $f^{\prime} / g^{\prime}$ is increasing on $(a, b)$, then $(f / g)^{\prime}<0$ on $(a, b)$.

(4) If $g g^{\prime}<0$ on $(a, b)$, limsup $\sup _{x \uparrow b} g^{2}(x)(f(x) / g(x))^{\prime} /\left|g^{\prime}(x)\right| \geq 0$ and $f^{\prime} / g^{\prime}$ is increasing on $(a, b)$, then $(f / g)^{\prime}>0$ on $(a, b)$.

Recently, Yang et al. [8], Theorem 1.2, established a more general monotonicity rule for the ratio of two power series.

Theorem 1.5 ([8]) Let $A(t)=\sum_{k=0}^{\infty} a_{k} t^{k}$ and $B(t)=\sum_{k=0}^{\infty} b_{k} t^{k}$ be two real power series converging on $(-r, r)$ and $b_{k}>0$ for all $k$, and $H_{A, B}=A^{\prime} B / B^{\prime}-A$. Suppose that for certain $m \in \mathbb{N}$, the non-constant sequence $\left\{a_{k} / b_{k}\right\}$ is increasing (decreasing) for $0 \leq k \leq m$ and decreasing (increasing) for $k \geq m$. Then the function $A / B$ is strictly increasing (decreasing) on $(0, r)$ if and only if $H_{A, B}\left(r^{-}\right) \geq(\leq) 0$. Moreover, if $H_{A, B}\left(r^{-}\right)<(>) 0$, then there exists $t_{0} \in(0, r)$ such that the function $A / B$ is strictly increasing (decreasing) on $\left(0, t_{0}\right)$ and strictly decreasing (increasing) on $\left(t_{0}, r\right)$.

The foregoing monotonicity rules have been used very effectively in the study of special functions [9-23], differential geometry [4, 24], probability [25] and approximation theory [26]. The main purpose of the article is to present the monotonicity rule for the function $\left[P(x)+\sum_{n=n_{0}}^{\infty} a_{n} x^{n}\right] /\left[P(x)+\sum_{n=n_{0}}^{\infty} b_{n} x^{n}\right]$ and to provide new bounds for the complete elliptic integral of the second kind. Some complicated computations are carried out using Mathematica computer algebra system.

\section{Monotonicity rule}

Theorem 2.1 Let $P(x)$ be a positive differentiable and decreasing function defined on $(0, r)$ $(r>0)$, let $A(x)=\sum_{n=n_{0}}^{\infty} a_{n} x^{n}$ and $B(x)=\sum_{n=n_{0}}^{\infty} b_{n} x^{n}$ be two real power series converging on 
$(-r, r)$. If $a_{n_{0}} / b_{n_{0}} \geq(\leq) 1, b_{n}>0$ for all $n \geq n_{0}$ and the non-constant sequence $\left\{a_{n} / b_{n}\right\}_{n=n_{0}}^{\infty}$ is increasing (decreasing), then the function $x \mapsto[P(x)+A(x)] /[P(x)+B(x)]$ is strictly increasing (decreasing) on $(0, r)$.

Proof Let $x \in(0, r)$, and

$$
\begin{aligned}
& I_{1}=\sum_{n=n_{0}}^{\infty}\left[n P(x)-x P^{\prime}(x)\right]\left(a_{n}-b_{n}\right) x^{n-1}, \\
& I_{2}=\sum_{n=n_{0}}^{\infty} n a_{n} x^{n-1} \sum_{n=n_{0}}^{\infty} b_{n} x^{n}-\sum_{n=n_{0}}^{\infty} a_{n} x^{n} \sum_{n=n_{0}}^{\infty} n b_{n} x^{n-1} .
\end{aligned}
$$

Then differentiating $[P(x)+A(x)] /[P(x)+B(x)]$ gives

$$
\begin{aligned}
{[P(x)} & +B(x)]^{2}\left[\frac{P(x)+A(x)}{P(x)+B(x)}\right]^{\prime} \\
= & \left(P^{\prime}(x)+\sum_{n=n_{0}}^{\infty} n a_{n} x^{n-1}\right)\left(P(x)+\sum_{n=n_{0}}^{\infty} b_{n} x^{n}\right) \\
& -\left(P(x)+\sum_{n=n_{0}}^{\infty} a_{n} x^{n}\right)\left(P^{\prime}(x)+\sum_{n=n_{0}}^{\infty} n b_{n} x^{n-1}\right) \\
= & I_{1}+I_{2} .
\end{aligned}
$$

Note that $I_{2}$ can be rewritten as

$$
\begin{aligned}
I_{2} & =\sum_{j=n_{0}}^{\infty} j a_{j} x^{j-1} \sum_{i=n_{0}}^{\infty} b_{i} x^{i}-\sum_{i=n_{0}}^{\infty} a_{i} x^{i} \sum_{j=n_{0}}^{\infty} j b_{j} x^{j-1} \\
& =\sum_{i=n_{0}}^{\infty} \sum_{j=n_{0}}^{\infty} j b_{i} b_{j}\left(\frac{a_{j}}{b_{j}}-\frac{a_{i}}{b_{i}}\right) x^{i+j-1} \\
& =\sum_{j=n_{0}}^{\infty} \sum_{i=n_{0}}^{\infty} i b_{j} b_{i}\left(\frac{a_{i}}{b_{i}}-\frac{a_{j}}{b_{j}}\right) x^{i+j-1} \\
& =\frac{1}{2} \sum_{i=n_{0}}^{\infty} \sum_{j=n_{0}}^{\infty} b_{i} b_{j}(i-j)\left(\frac{a_{i}}{b_{i}}-\frac{a_{j}}{b_{j}}\right) x^{i+j-1} .
\end{aligned}
$$

If $a_{n_{0}} / b_{n_{0}} \geq(\leq) 1, b_{n}>0$ for all $n \geq n_{0}$ and the non-constant sequence $\left\{a_{n} / b_{n}\right\}_{n=n_{0}}^{\infty}$ is increasing (decreasing), then we clearly see that

$$
a_{n} \geq(\leq) b_{n}
$$

for all $n \geq n_{0}$ and

$$
\sum_{i=n_{0}}^{\infty} \sum_{j=n_{0}}^{\infty} b_{i} b_{j}(i-j)\left(\frac{a_{i}}{b_{i}}-\frac{a_{j}}{b_{j}}\right) x^{i+j-1}>(<) 0
$$

for all $x \in(0, r)$. 
It follows from $P(x)$ is a positive differentiable and decreasing function on $(0, r)$ that

$$
n P(x)-x P^{\prime}(x)>0
$$

for all $x \in(0, r)$.

Therefore, $[(P(x)+A(x)) /(P(x)+B(x))]^{\prime}>(<) 0$ for all $x \in(0, r)$ follows easily from (2.1)(2.6), and the proof of Theorem 2.1 is completed.

\section{Bounds for the complete elliptic integral of the second kind}

For $r \in(0,1)$, Legendre's complete elliptic integral [27] of the second kind is given by

$$
\mathcal{E}(r)=\int_{0}^{\pi / 2} \sqrt{1-r^{2} \sin ^{2}(t)} d t .
$$

It is well known that $\mathcal{E}\left(0^{+}\right)=\pi / 2, \mathcal{E}\left(1^{-}\right)=1$, and $\mathcal{E}(r)$ is the particular case of the Gaussian hypergeometric function

$$
F(a, b ; c ; x)=\sum_{n=0}^{\infty} \frac{(a)_{n}(b)_{n}}{(c)_{n}} \frac{x^{n}}{n !} \quad(-1<x<1)
$$

where $(a)_{n}=\Gamma(a+n) / \Gamma(a)$ and $\Gamma(x)=\int_{0}^{\infty} t^{x-1} e^{-t} d t(x>0)$ is the gamma function. Indeed, we have

$$
\mathcal{E}(r)=\frac{\pi}{2} F\left(-\frac{1}{2}, \frac{1}{2} ; 1 ; r^{2}\right)=\frac{\pi}{2} \sum_{n=0}^{\infty} \frac{\left(-\frac{1}{2}\right)_{n}\left(\frac{1}{2}\right)_{n}}{(n !)^{2}} r^{2 n}
$$

Recently, the bounds for the complete elliptic integral $\mathcal{E}(r)$ of the second kind have been the subject of intensive research. In particular, many remarkable inequalities for $\mathcal{E}(r)$ can be found in the literature [28-41]. Vuorinen [42] conjectured that the inequality

$$
\mathcal{E}(r) \geq \frac{\pi}{2}\left(\frac{1+r^{\prime 3 / 2}}{2}\right)^{2 / 3}
$$

holds for all $r \in(0,1)$, where, and in what follows, $r^{\prime}=\left(1-r^{2}\right)^{1 / 2}$. Inequality (3.2) was proved by Barnard et al. in [43].

Very recently, the accurate bounds for $\mathcal{E}(r)$ in terms of the Stolarsky mean $S_{p, q}\left(1, r^{\prime}\right)$ were given in $[44,45]$ :

$$
\begin{aligned}
& \frac{\pi}{2} S_{11 / 4,7 / 4}\left(1, r^{\prime}\right)<\mathcal{E}(r)<\frac{11}{7} S_{11 / 4,7 / 4}\left(1, r^{\prime}\right), \\
& \frac{25}{16} S_{5 / 2,2}\left(1, r^{\prime}\right)<\mathcal{E}(r)<\frac{\pi}{2} S_{5 / 2,2}\left(1, r^{\prime}\right),
\end{aligned}
$$

where $S_{p, q}(a, b)=\left[q\left(a^{p}-b^{p}\right) /\left(p\left(a^{q}-b^{q}\right)\right)\right]^{1 /(p-q)}$.

In this section, we shall use Theorem 2.1 to present new bounds for the complete elliptic integral $\mathcal{E}(r)$ of the second kind. In order to prove our main result, we need three lemmas, which we present in this section. 
Lemma 3.1 (see [46], Lemma 7) Let $n \in \mathbb{N}$ and $m \in \mathbb{N} \cup\{0\}$ with $n>m, a_{i} \geq 0$ for all $0 \leq i \leq n, a_{n} a_{m}>0$ and

$$
P_{n}(t)=-\sum_{i=0}^{m} a_{i} t^{i}+\sum_{i=m+1}^{n} a_{i} t^{i} .
$$

Then there exists $t_{0} \in(0, \infty)$ such that $P_{n}\left(t_{0}\right)=0, P_{n}(t)<0$ for $t \in\left(0, t_{0}\right)$ and $P_{n}(t)>0$ for $t \in\left(t_{0}, \infty\right)$.

Lemma 3.2 (see $[47,48]$ ) The double inequality

$$
\frac{1}{(x+a)^{1-a}}<\frac{\Gamma(x+a)}{\Gamma(x+1)}<\frac{1}{x^{1-a}}
$$

holds for all $x>0$ and $a \in(0,1)$.

Lemma 3.3 Let $n \in \mathbb{N}, p_{1}(n), p_{2}(n), p_{3}(n)$ and $w_{n}$ be defined by

$$
\begin{aligned}
& p_{1}(n)= 125 n^{4}+1,482 n^{3}+1,601 n^{2}-7,532 n+1,944, \\
& p_{2}(n)= 4,375 n^{6}+213,247 n^{5}+1,696,697 n^{4}-4,433,167 n^{3} \\
&-1,571,832 n^{2}+4,662,360 n-1,555,200, \\
& p_{3}(n)= 625 n^{6}+22,583 n^{5}-304,413 n^{4}+234,181 n^{3} \\
&+1,906,328 n^{2}-2,918,064 n+675,360, \\
& w_{n}=-648 n p_{1}(n)\left(\frac{1}{4}\right)_{n-2}+p_{2}(n)\left(\frac{1}{2}\right)_{n-3}+\frac{5 n p_{3}(n)}{2 n-5}\left(\frac{3}{4}\right)_{n-3},
\end{aligned}
$$

respectively. Then $w_{n} \geq 0$ for all $n \geq 3$.

Proof Let $p_{0}(n), p_{4}(n), p_{5}(n), \alpha_{n}$ and $\beta_{n}$ be defined by

$$
\begin{aligned}
& p_{0}(n)= 625 n^{4}-2,018 n^{3}+3,985 n^{2}-4,032 n+1,080, \\
& p_{4}(n)= 875 n^{7}-81,128 n^{6}-2,341,894 n^{5}-10,120,928 n^{4}+19,839,719 n^{3} \\
&-22,615,904 n^{2}+73,667,340 n-71,971,200, \\
& p_{5}(n)= 250 n^{6}-1,834 n^{5}+391,275 n^{4}+975,000 n^{3}-7,770,415 n^{2} \\
&+7,980,844 n+298,140, \\
& \alpha_{n}=\frac{p_{4}(n)}{n(n+1)}\left(\frac{1}{2}\right)_{n-3}, \\
& \beta_{n}=\frac{10(n-1) p_{5}(n)}{(2 n-3)(2 n-5)}\left(\frac{3}{4}\right)_{n-3},
\end{aligned}
$$

respectively.

Then from (3.5)-(3.13) and elaborated computations we get

$$
w_{3}=w_{4}=w_{5}=w_{6}=0,
$$




$$
\begin{aligned}
& w_{7}=\frac{2,848,355}{16}>0 \text {, } \\
& \frac{w_{n+1}}{(n+1) p_{1}(n+1)}-\frac{(n-7 / 4) w_{n}}{n p_{1}(n)} \\
& =\frac{p_{2}(n+1)}{(n+1) p_{1}(n+1)}\left(\frac{1}{2}\right)_{n-2}-\frac{(n-7 / 4) p_{2}(n)}{n p_{1}(n)}\left(\frac{1}{2}\right)_{n-3} \\
& +\frac{5 p_{3}(n+1)}{(2 n-3) p_{1}(n+1)}\left(\frac{3}{4}\right)_{n-2}-\frac{5(n-7 / 4) p_{3}(n)}{(2 n-5) p_{1}(n)}\left(\frac{3}{4}\right)_{n-3} \\
& =\left[\frac{(n-5 / 2) p_{2}(n+1)}{(n+1) p_{1}(n+1)}-\frac{(n-7 / 4) p_{2}(n)}{n p_{1}(n)}\right]\left(\frac{1}{2}\right)_{n-3} \\
& +\left[\frac{5(n-9 / 4) p_{3}(n+1)}{(2 n-3) p_{1}(n+1)}-\frac{5(n-7 / 4) p_{3}(n)}{(2 n-5) p_{1}(n)}\right]\left(\frac{3}{4}\right)_{n-3} \\
& =\frac{p_{0}(n+1) p_{4}(n)}{4 n(n+1) p_{1}(n) p_{1}(n+1)}\left(\frac{1}{2}\right)_{n-3} \\
& +\frac{5(n-1) p_{0}(n+1) p_{5}(n)}{2(2 n-3)(2 n-5) p_{1}(n) p_{1}(n+1)}\left(\frac{3}{4}\right)_{n-3} \\
& =\frac{p_{0}(n+1)}{4 p_{1}(n) p_{1}(n+1)}\left(\alpha_{n}+\beta_{n}\right) \\
& \frac{\alpha_{n}}{\beta_{n}}=\frac{(2 n-3)(2 n-5) p_{4}(n)}{10 n(n-1)(n+1) p_{5}(n)} \frac{\left(\frac{1}{2}\right)_{n-3}}{\left(\frac{3}{4}\right)_{n-3}} \\
& \frac{\alpha_{7}}{\beta_{7}}=-\frac{7,313,056}{7,313,875} \\
& \frac{10\left(\frac{3}{4}\right)_{n-3}}{\left(\frac{1}{2}\right)_{n-1}}\left(\frac{\alpha_{n+1}}{\beta_{n+1}}-\frac{\alpha_{n}}{\beta_{n}}\right) \\
& =\frac{(2 n-1)(2 n-3)(n-5 / 2) p_{4}(n+1)}{n(n+1)(n+2)(n-9 / 4) p_{5}(n+1)}-\frac{(2 n-3)(2 n-5) p_{4}(n)}{n(n-1)(n+1) p_{5}(n)} \\
& =-\frac{(2 n-3)(2 n-5)(n-3)(n-4)\left(125 n^{4}+1,982 n^{3}+6,797 n^{2}+616 n-2,380\right)}{n(4 n-9)(n-1)(n+1)(n+2) p_{5}(n) p_{5}(n+1)} \\
& \times\left(1,750 n^{8}-802,264 n^{7}-54,353,513 n^{6}+811,227,431 n^{5}-4,748,597,075 n^{4}\right. \\
& +21,568,863,989 n^{3}-80,185,046,202 n^{2} \\
& +144,028,552,644 n-85,225,499,160) \\
& =-\frac{(2 n-3)(2 n-5)(n-3)(n-4)\left(125 n^{4}+1,982 n^{3}+6,797 n^{2}+616 n-2,380\right)}{n(4 n-9)(n-1)(n+1)(n+2) p_{5}(n) p_{5}(n+1)} \\
& \times\left[-420,331,641,120-714,515,222,844(n-7)-475,749,995,856(n-7)^{2}\right. \\
& -152,526,681,341(n-7)^{3}-25,642,525,865(n-7)^{4} \\
& -2,263,535,771(n-7)^{5} \\
& \left.-91,263,449(n-7)^{6}-704,264(n-7)^{7}+1,750(n-7)^{8}\right] .
\end{aligned}
$$

From Lemma 3.1 and (3.19) together with the facts that $p_{5}(n)>0$ and $p_{5}(n+1)>0$ for $n \geq 7$, we clearly see that there exists $n_{0}>7$ such that the sequence $\left\{\alpha_{n} / \beta_{n}\right\}_{n=7}^{\infty}$ is increasing 
for $7 \leq n \leq n_{0}$ and decreasing for $n \geq n_{0}$, which implies that

$$
\frac{\alpha_{n}}{\beta_{n}} \geq \min \left\{\frac{\alpha_{7}}{\beta_{7}}, \lim _{n \rightarrow \infty} \frac{\alpha_{n}}{\beta_{n}}\right\}
$$

It follows from Lemma 3.2 that

$$
\frac{\Gamma\left(\frac{3}{4}\right)\left(n-\frac{5}{2}\right)^{-1 / 4}}{\Gamma\left(\frac{1}{2}\right)}<\frac{\left(\frac{1}{2}\right)_{n-3}}{\left(\frac{3}{4}\right)_{n-3}}=\frac{\Gamma\left(\frac{3}{4}\right)}{\Gamma\left(\frac{1}{2}\right)} \frac{\Gamma\left(n-\frac{13}{4}+\frac{3}{4}\right)}{\Gamma\left(n-\frac{13}{4}+1\right)}<\frac{\Gamma\left(\frac{3}{4}\right)\left(n-\frac{13}{4}\right)^{-1 / 4}}{\Gamma\left(\frac{1}{2}\right)}
$$

for all $n \geq 7$.

From (3.10), (3.11), (3.17), (3.18), (3.20) and (3.21) we get

$$
\frac{\alpha_{n}}{\beta_{n}} \geq \frac{\alpha_{7}}{\beta_{7}}>-1
$$

for all $n \geq 7$.

Therefore, Lemma 3.3 follows easily from (3.14)-(3.16) and (3.22) together with the facts that $p_{1}(n)>0, p_{0}(n+1)>0$ and $p_{1}(n+1)>0$ for $n \geq 7$.

Theorem 3.4 The double inequality

$$
\frac{40(\pi-2)}{29} J\left(r^{\prime}\right)-\frac{51 \pi-160}{58}<\mathcal{E}(r)<\frac{\pi}{2} J\left(r^{\prime}\right)
$$

holds for all $r \in(0,1)$, where

$$
J\left(r^{\prime}\right)=\frac{51 r^{\prime 2}+20 r^{\prime} \sqrt{r^{\prime}}+50 r^{\prime}+20 \sqrt{r^{\prime}}+51}{16\left(5 r^{\prime}+2 \sqrt{r^{\prime}}+5\right)} .
$$

Proof Let $r \in(0,1), x=r^{2}, P(x), f_{1}(r), f_{2}(r)$ and $F(r)$ be defined by

$$
\begin{aligned}
P(x)= & 1,536-1,248 x, \\
f_{1}(r)= & 2,592\left(2-r^{2}\right)\left(1-r^{2}\right)^{3 / 4}+15\left(425 r^{4}-624 r^{2}+192\right)\left(1-r^{2}\right)^{1 / 2} \\
& -50\left(r^{4}-96 r^{2}+96\right)\left(1-r^{2}\right)^{1 / 4}-105 r^{4}+3,600 r^{2}+2,880, \\
f_{2}(r)= & 625 r^{4}-384 r^{2}+384, \\
F(r)= & \frac{1-J\left(r^{\prime}\right)}{1-2 \mathcal{E}(r) / \pi},
\end{aligned}
$$

respectively.

Then from (3.1) and (3.24)-(3.28) we have

$$
\begin{aligned}
f_{1}(r)= & 2,880+3,600 r^{2}-105 r^{4}+2,592\left(2 \sum_{n=0}^{\infty} \frac{\left(-\frac{3}{4}\right)_{n}}{n !} r^{2 n}-\sum_{n=1}^{\infty} \frac{\left(-\frac{3}{4}\right)_{n-1}}{(n-1) !} r^{2 n}\right) \\
& +15\left(425 \sum_{n=2}^{\infty} \frac{\left(-\frac{1}{2}\right)_{n-2}}{(n-2) !} r^{2 n}-624 \sum_{n=1}^{\infty} \frac{\left(-\frac{1}{2}\right)_{n-1}}{(n-1) !} r^{2 n}+192 \sum_{n=0}^{\infty} \frac{\left(-\frac{1}{2}\right)_{n}}{(n !} r^{2 n}\right)
\end{aligned}
$$




$$
\begin{aligned}
& -50\left(\sum_{n=2}^{\infty} \frac{\left(-\frac{1}{4}\right)_{n-2}}{(n-2) !} r^{2 n}-96 \sum_{n=1}^{\infty} \frac{\left(-\frac{1}{4}\right)_{n-1}}{(n-1) !} r^{2 n}+96 \sum_{n=0}^{\infty} \frac{\left(-\frac{1}{4}\right)_{n}}{n !} r^{2 n}\right) \\
= & 6,144-7,680 r^{2}-105 r^{4}+2,592 \sum_{n=2}^{\infty} \frac{\left(n-\frac{7}{2}\right)\left(-\frac{3}{4}\right)_{n-1}}{n !} r^{2 n} \\
& -15 \sum_{n=2}^{\infty} \frac{\left(7 n^{2}-367 n-720\right)\left(-\frac{1}{2}\right)_{n-2}}{n !} r^{2 n} \\
& -50 \sum_{n=2}^{\infty} \frac{\left(n^{2}-121 n+270\right)\left(-\frac{1}{4}\right)_{n-2}}{n !} r^{2 n} \\
= & 6,144-7,680 r^{2}+11,248 r^{4}-1,944 \sum_{n=3}^{\infty} \frac{\left(n-\frac{7}{2}\right)\left(\frac{1}{4}\right)_{n-2}}{n !} r^{2 n} \\
& +\frac{15}{2} \sum_{n=3}^{\infty} \frac{\left(7 n^{2}-367 n-720\right)\left(\frac{1}{2}\right)_{n-3}}{n !} r^{2 n} \\
& +\frac{25}{2} \sum_{n=3}^{\infty} \frac{\left(n^{2}-121 n+270\right)\left(\frac{3}{4}\right)_{n-3}}{n !} r^{2 n}, \\
16 f_{2}(r) & -f_{1}(r) \\
=1,536 r^{2}-1,248 r^{4}-\frac{25}{2} \sum_{n=3}^{\infty} \frac{\left(n^{2}-121 n+270\right)\left(\frac{3}{4}\right)_{n-3}}{n !} r^{2 n} & \\
- & \frac{15}{2} \sum_{n=3}^{\infty} \frac{\left(7 n^{2}-367 n-720\right)\left(\frac{1}{2}\right)_{n-3}}{n !} r^{2 n}+1,944 \sum_{n=3}^{\infty} \frac{\left(n-\frac{7}{2}\right)\left(\frac{1}{4}\right)_{n-2}}{n !} r^{2 n} \\
=1,536 r^{2}-1,248 r^{4}+\sum_{n=3}^{\infty} u_{n} r^{2 n}, &
\end{aligned}
$$

where

$$
\begin{aligned}
u_{n}= & \frac{972(2 n-7)}{n !}\left(\frac{1}{4}\right)_{n-2} \\
& -\frac{15\left(7 n^{2}-367 n-720\right)}{2 n !}\left(\frac{1}{2}\right)_{n-3}-\frac{25\left(n^{2}-121 n+270\right)}{2 n !}\left(\frac{3}{4}\right)_{n-3}, \\
1- & \frac{2}{\pi} \mathcal{E}(r) \\
= & -\sum_{n=1}^{\infty} \frac{\left(-\frac{1}{2}\right)_{n}\left(\frac{1}{2}\right)_{n}}{(n !)^{2}} r^{2 n}=\sum_{n=1}^{\infty} \frac{\left(\frac{1}{2}\right)_{n-1}\left(\frac{1}{2}\right)_{n}}{2(n !)^{2}} r^{2 n}, \\
16 f_{2}(r)\left(1-\frac{2}{\pi} \mathcal{E}(r)\right) & 8\left(625 r^{4}-384 r^{2}+384\right) \sum_{n=1}^{\infty} \frac{\left(\frac{1}{2}\right)_{n-1}\left(\frac{1}{2}\right)_{n}}{(n !)^{2}} r^{2 n} \\
= & 1,536 r^{2}-1,248 r^{4}+\sum_{n=3}^{\infty} v_{n} r^{2 n},
\end{aligned}
$$


where

$$
v_{n}=\frac{8 p_{0}(n)}{(n !)^{2}}\left(\frac{1}{2}\right)_{n-3}\left(\frac{1}{2}\right)_{n-2}
$$

and $p_{0}(n)$ is defined by (3.9).

$$
\begin{aligned}
J\left(r^{\prime}\right) & =\frac{\left(51 r^{\prime 2}+20 r^{\prime} \sqrt{r^{\prime}}+50 r^{\prime}+20 \sqrt{r^{\prime}}+51\right)\left(5 r^{\prime}-2 \sqrt{r^{\prime}}+5\right)\left(25 r^{\prime 2}-46 r^{\prime}+25\right)}{16\left(5 r^{\prime}+2 \sqrt{r^{\prime}}+5\right)\left(5 r^{\prime}-2 \sqrt{r^{\prime}}+5\right)\left(25 r^{\prime 2}-46 r^{\prime}+25\right)} \\
& =\frac{f_{1}(r)}{16 f_{2}(r)}, \\
F(r) & =\frac{16 f_{2}(r)-f_{1}(r)}{16 f_{2}(r)\left(1-\frac{2}{\pi} \mathcal{E}(r)\right)}=\frac{P(x)+\sum_{n=2}^{\infty} u_{n+1} x^{n}}{P(x)+\sum_{n=2}^{\infty} v_{n+1} x^{n}} .
\end{aligned}
$$

It follows from (3.9), (3.29), (3.30) and elaborated computations that

$$
\begin{aligned}
& \frac{u_{3}}{v_{3}}=1, \\
& u_{n+1}-\frac{v_{n+1}}{v_{n}} u_{n}=-\frac{15(2 n-5)}{8(n+1) p_{0}(n)(n+1) !} w_{n},
\end{aligned}
$$

where $w_{n}$ is defined by (3.8).

It is not difficult to verify that

$$
p_{0}(n)=625 n^{4}-2,018 n^{3}+3,985 n^{2}-4,032 n+1,080>0
$$

for all $n \geq 3$.

From Lemma 3.3, (3.30), (3.33) and (3.34) we know that

$$
v_{n}>0
$$

for all $n \geq 3$, and the sequence $\left\{u_{n} / v_{n}\right\}_{n=3}^{\infty}$ is decreasing.

Equation (3.25) implies that

$$
P(x)>0
$$

for $x \in(0,1)$, and $P(x)$ is decreasing on $(0,1)$.

It follows from Theorem 2.1, (3.31) and (3.32) together with the monotonicity of the sequence $\left\{u_{n} / v_{n}\right\}_{n=3}^{\infty}$ and the function $P(x)$ on $(0,1)$ that the function $F(r)$ is strictly decreasing on $(0,1)$ and

$$
\lim _{r \rightarrow 1^{-}} F(r)<F(r)<\lim _{r \rightarrow 0^{+}} F(r)
$$

for all $r \in(0,1)$.

Note that (3.24), (3.28) and (3.31) lead to the conclusion that

$$
\lim _{r \rightarrow 1^{-}} F(r)=\frac{1-J\left(0^{+}\right)}{1-2 \mathcal{E}\left(1^{-}\right) / \pi}=\frac{1-51 / 80}{1-2 / \pi}=\frac{29 \pi}{80(\pi-2)}, \quad \lim _{r \rightarrow 0^{+}} F(r)=1 .
$$


Therefore, Theorem 3.4 follows from (3.28), (3.37) and (3.38).

Remark 3.5 Let

$$
\begin{array}{ll}
\lambda_{1}(r)=\frac{\pi}{2} S_{11 / 4,7 / 4}\left(1, r^{\prime}\right), & \mu_{1}(r)=\frac{11}{7} S_{11 / 4,7 / 4}\left(1, r^{\prime}\right), \\
\lambda_{2}(r)=\frac{25}{16} S_{5 / 2,2}\left(1, r^{\prime}\right), & \mu_{2}(r)=\frac{\pi}{2} S_{5 / 2,2}\left(1, r^{\prime}\right), \\
\lambda(r)=\frac{40(\pi-2)}{29} J\left(r^{\prime}\right)-\frac{51 \pi-160}{58}, & \mu(r)=\frac{\pi}{2} J\left(r^{\prime}\right),
\end{array}
$$

where $J\left(r^{\prime}\right)$ is defined by (3.24). Then simple computations lead to

$$
\begin{aligned}
& \lambda_{1}\left(1^{-}\right)=\frac{7 \pi}{22}=0.999597 \ldots, \quad \mu_{1}\left(0^{+}\right)=\frac{11}{7}=1.571428 \ldots, \\
& \lambda_{2}\left(0^{+}\right)=\frac{25}{16}=1.5625, \quad \mu_{2}\left(1^{-}\right)=\frac{2 \pi}{5}=1.256637 \ldots, \\
& \lambda\left(0^{+}\right)=\frac{\pi}{2}=\mu\left(0^{+}\right)=1.5707963 \ldots, \\
& \lambda\left(1^{-}\right)=1, \quad \mu\left(1^{-}\right)=\frac{51 \pi}{160}=1.00138 \ldots .
\end{aligned}
$$

From (3.3), (3.4), (3.23) and (3.39)-(3.45) we clearly see that there exists small enough $\delta \in(0,1)$ such that the lower bound given in (3.23) for $\mathcal{E}(r)$ is better than the lower bound given in (3.3) for $r \in(\delta, 1-\delta)$, the lower bound given in (3.23) for $\mathcal{E}(r)$ is better than the lower bound given in (3.4) for $r \in(0, \delta)$, the upper bound given in (3.23) for $\mathcal{E}(r)$ is better than the upper bound given in (3.3) for $r \in(0, \delta)$, and the upper bound given in (3.23) for $\mathcal{E}(r)$ is better than the upper bound given in (3.4) for $r \in(\delta, 1-\delta)$.

Corollary 3.6 Let $J\left(r^{\prime}\right)$ be defined by (3.24). Then the double inequality

$$
\frac{\pi}{2} J\left(r^{\prime}\right)-\left(\frac{51 \pi}{160}-1\right)<\mathcal{E}(r)<\frac{\pi}{2} J\left(r^{\prime}\right)
$$

holds for all $r \in(0,1)$.

Proof Let $F(r)$ be defined by (3.28) and

$$
A(r)=J\left(r^{\prime}\right)-\frac{2}{\pi} \mathcal{E}(r)
$$

Then we clearly see that

$$
\begin{aligned}
& A\left(0^{+}\right)=J\left(1^{-}\right)-\frac{2}{\pi} \mathcal{E}\left(0^{+}\right)=0, \quad A\left(1^{-}\right)=J\left(0^{+}\right)-\frac{2}{\pi} \mathcal{E}\left(1^{-}\right)=\frac{51 \pi-160}{80 \pi}, \\
& A(r)=\left[1-\frac{2}{\pi} \mathcal{E}(r)\right]\left[1-\frac{1-J\left(r^{\prime}\right)}{1-\frac{2}{\pi} \mathcal{E}(r)}\right]=\left[1-\frac{2}{\pi} \mathcal{E}(r)\right][1-F(r)] .
\end{aligned}
$$

From (3.49) and the proof of Theorem 3.4 we know that $F(r)$ is strictly decreasing on $(0,1)$ and $A(r)$ is strictly increasing on $(0,1)$. Therefore, inequality (3.46) follows from (3.47) and (3.48) together with the monotonicity of $A(r)$ on the interval $(0,1)$. 
Corollary 3.7 Let $J\left(r^{\prime}\right)$ be defined by (3.24). Then the double inequality

$$
\frac{80}{51} J\left(r^{\prime}\right)<\mathcal{E}(r)<\frac{\pi}{2} J\left(r^{\prime}\right)
$$

holds for all $r \in(0,1)$.

Proof Let $A(r)$ be defined by (3.47) and

$$
B(r)=\frac{J\left(r^{\prime}\right)}{\frac{2}{\pi} E(r)}
$$

Then we clearly see that

$$
\begin{aligned}
& B\left(0^{+}\right)=\frac{J\left(1^{-}\right)}{\frac{2}{\pi} E\left(0^{+}\right)}=1, \quad B\left(1^{-}\right)=\frac{J\left(0^{+}\right)}{\frac{2}{\pi} E\left(1^{-}\right)}=\frac{51 \pi}{160}, \\
& B(r)=\frac{1}{\frac{2}{\pi} \mathcal{E}(r)}\left[J\left(r^{\prime}\right)-\frac{2}{\pi} \mathcal{E}(r)\right]+1=\frac{A(r)}{\frac{2}{\pi} \mathcal{E}(r)}+1 .
\end{aligned}
$$

From (3.53) and the proof of Corollary 3.6 we know that both $A(r)$ and $B(r)$ are strictly increasing on $(0,1)$. Therefore, inequality (3.50) follows from (3.51) and (3.52) together with the monotonicity of $B(r)$ on the interval $(0,1)$.

Remark 3.8 From Corollaries 3.6 and 3.7 we have

$$
\left|\mathcal{E}(r)-\frac{\pi}{2} J\left(r^{\prime}\right)\right|<\frac{51 \pi}{160}-1=0.001382 \ldots, \quad\left|\frac{\mathcal{E}(r)-\frac{\pi}{2} J\left(r^{\prime}\right)}{\mathcal{E}(r)}\right|<\frac{51 \pi}{160}-1=0.001382 \ldots
$$

for all $r \in(0,1)$, which implies that both the absolute and relative errors using $\pi J\left(r^{\prime}\right) / 2$ to approximate $\mathcal{E}(r)$ are less than $0.14 \%$.

\section{Conclusions}

In this paper, we find a monotonicity rule for the function $\left[P(x)+\sum_{n=n_{0}}^{\infty} a_{n} x^{n}\right] /[P(x)+$ $\left.\sum_{n=n_{0}}^{\infty} b_{n} x^{n}\right]$. As applications, we present new bounds for the complete elliptic integral $\mathcal{E}(r)=\int_{0}^{\pi / 2} \sqrt{1-r^{2} \sin ^{2} t} d t(0<r<1)$ of the second kind, and we show that our bounds are sharper than the previously known bounds for some $r \in(0,1)$.

Competing interests

The authors declare that they have no competing interests.

Authors' contributions

All authors contributed equally to the writing of this paper. All authors read and approved the final manuscript.

Author details

'Department of Mathematics, Huzhou University, Huzhou, 313000, China. ${ }^{2}$ School of Distance Education, Huzhou

Broadcast and TV University, Huzhou, 313000, China. ${ }^{3}$ Friedman Brain Institute, Icahn School of Medicine at Mount Sinai, New York, 10029, USA.

\section{Acknowledgements}

The research was supported by the Natural Science Foundation of China (Grants Nos. 61673169, 61374086, 11371125, 11401191), the Tianyuan Special Funds of the National Natural Science Foundation of China (Grant No. 11626101) and the Natural Science Foundation of the Department of Education of Zhejiang Province (Grant No. Y201635325). 


\section{Publisher's Note}

Springer Nature remains neutral with regard to jurisdictional claims in published maps and institutional affiliations.

\section{Received: 17 March 2017 Accepted: 26 April 2017 Published online: 09 May 2017}

\section{References}

1. Biernacki, M, Krzyż, J: On the monotonicity of certain functionals in the theory of analytic functions. Ann. Univ. Mariae Curie-Skłodowska, Sect. A 9, 135-147 (1955)

2. Ponnusamy, S, Vuorinen, M: Asymptotic expansions and inequalities for hypergeometric functions. Mathematika 44(2), 278-301 (1997)

3. Heikkala, V, Vamanamurthy, MK, Vuorinen, M: Generalized elliptic integrals. Comput. Methods Funct. Theory 9(1), 75-109 (2009)

4. Cheeger, J, Gromov, M, Taylor, M: Finite propagation speed, kernel estimates for functions of the Laplace operator, and the geometry of complete Riemannian manifolds. J. Differ. Geom. 17(1), 15-53 (1982)

5. Anderson, GD, Vamanamurthy, MK, Vuorinen, M: Inequalities for quasiconformal mappings in space. Pac. J. Math. $160(1), 1-18(1993)$

6. Anderson, GD, Vamanamurthy, MK, Vuorinen, M: Conformal Invariants, Inequalities, and Quasiconformal Maps. John Wiley \& Sons, New York (1997)

7. Pinelis, I: L'Hospital type rules for oscillation, with applications. JIPAM. J. Inequal. Pure Appl. Math. 2(3), Article ID 33 (2001)

8. Yang, Z-H, Chu, Y-M, Wang, M-K: Monotonicity criterion for the quotient of power series with applications. J. Math. Anal. Appl. 428(1), 587-604 (2015)

9. Balasubramanian, R, Ponnusamy, S, Vuorinen, M: Functional inequalities for the quotients of hypergeometric functions. J. Math. Anal. Appl. 218(1), 256-268 (1998)

10. Anderson, GD, Qiu, S-L, Vamanamurthy, MK, Vuorinen, M: Generalized elliptic integrals and modular equations. Pac. J. Math. 192(1), 1-37 (2000)

11. Anderson, GD, Vamanamurthy, MK, Vuorinen, M: Topics in special functions. In: Papers on Analysis. Rep. Univ. Jyväskylä Dep. Math. Stat., vol. 83, pp. 5-26. Univ. Jyväskylä, Jyväskylä (2001)

12. Alzer, H, Qiu, S-L: Monotonicity theorems and inequalities for the complete elliptic integrals. J. Comput. Appl. Math. 172(2), 289-312 (2004)

13. Baricz, A: Landen-type inequality for Bessel functions. Comput. Methods Funct. Theory 5(2), 373-379 (2005)

14. Zhu, L: Sharpening Jordan's inequality and the Yang Le inequality. Appl. Math. Lett. 19(3), 240-243 (2006)

15. Anderson, GD, Vamanamurthy, MK, Vuorinen, M: Generalized convexity and inequalities. J. Math. Anal. Appl. 355(2), 1294-1308 (2007)

16. Zhu, L: New inequalities for hyperbolic functions and their applications. J. Inequal. Appl. 2012, Article ID 303 (2012)

17. Yang, Z-H: New sharp bounds for identric mean in terms of logarithmic mean and arithmetic mean. J. Math. Inequal. 6(4), 533-543 (2012)

18. Yang, Z-H, Jiang, Y-L, Song, Y-Q, Chu, Y-M: Sharp inequalities for trigonometric functions. Abstr. Appl. Anal. 2014 Article ID 601839 (2014)

19. Baricz, Á: Landen inequalities for special functions. Proc. Am. Math. Soc. 142(9), 3059-3066 (2014)

20. Wang, MK, Chu, Y-M, Song, Y-Q: Asymptotical formulas for Gaussian and generalized hypergeometric functions. Appl. Math. Comput. 276, 44-60 (2016)

21. Yang, Z-H, Chu, Y-M: On approximating the modified Bessel function of the second kind. J. Inequal. Appl. 2017, Article ID 41 (2017)

22. Wang, $\mathrm{M}-\mathrm{K}, \mathrm{Chu}, \mathrm{Y}-\mathrm{M}$ : Refinements of transformation inequalities for zero-balanced hypergeometric functions. Acta Math. Sci. 37B(3), 607-622 (2017)

23. Wang, M-K, Li, Y-M, Chu, Y-M: Inequalities and infinite product formula for Ramanujan generalized modular equation function. Ramanujan J. doi:10.1007/s11139-017-9888-3

24. Chavel, I: Riemannian Geometry - A Modern Introduction. Cambridge University Press, Cambridge (1993)

25. Pinelis, I: L'Hospital type rules for monotonicity: applications to probability inequalities for sums of bounded random variables. J. Inequal. Pure Appl. Math. 3(1), Article ID 7 (2002)

26. Pinelis, I: Monotonicity properties of the relative error of a Padé approximation for Mills ratio. JIPAM. J. Inequal. Pure Appl. Math. 3(2), Article ID 20 (2002)

27. Abramowitz, M, Stegun, IA: Handbook of Mathematical Functions with Formulas, Graphs, and Mathematical Tables. U. S. Government Printing Office, Washington (1964)

28. Anderson, GD, Vamanamurthy, MK, Vuorinen, M: Functional inequalities for hypergeometric functions and complete elliptic integrals. SIAM J. Math. Anal. 23(2), 512-524 (1992)

29. Guo, B-N, Qi, F: Some bounds for the complete elliptic integrals of the first and second kinds. Math. Inequal. Appl. 14(2), 323-334 (2001)

30. Chu, Y-M, Wang, M-K, Qiu, S-L: Optimal combinations bounds of root-square and arithmetic means for Toader mean. Proc. Indian Acad. Sci. Math. Soc. 122(1), 41-51 (2012)

31. Chu, Y-M, Wang, M-K: Optimal Lehmer mean bounds for the Toader mean. Results Math. 61(3-4), 223-229 (2012)

32. Chu, Y-M, Wang, M-K, Qiu, S-L, Jiang, Y-P: Bounds for complete elliptic integrals of the second kind with applications. Comput. Math. Appl. 63(7), 1177-1184 (2012)

33. Wang, M-K, Chu, Y-M, Qiu, S-L, Jiang, Y-P: Bounds for the perimeter of an ellipse. J. Approx. Theory 164(7), 928-937 (2012)

34. Wang, M-K, Chu, Y-M: Asymptotical bounds for the complete elliptic integrals of the second kind. J. Math. Anal. Appl. 402(1), 119-126 (2013)

35. Song, Y-Q, Jiang, W-D, Chu, Y-M, Yan, D-D: Optimal bounds for Toader mean in terms of arithmetic and contraharmonic means. J. Math. Inequal. 7(4), 751-757 (2013)

36. Wang, M-K, Chu, Y-M, Jiang, Y-P, Qiu, S-L: Bounds of the perimeter of an ellipse using arithmetic, geometric and harmonic means. Math. Inequal. Appl. 17(1), 101-111 (2014) 
37. Li, Y, Qi, F: Some inequalities for complete elliptic integrals. Appl. Math. E-Notes 14, 193-199 (2014)

38. Hua, Y, Qi, F: The best bounds for Toader mean in terms of the centroidal and arithmetic means. Filomat 28(4), 775-780 (2014)

39. Hua, Y, Qi, F: A double inequality for bounding Toader mean by the centroidal mean. Proc. Indian Acad. Sci. Math. Sci. 124(4), 527-531 (2014)

40. Jiang, W-D, Qi, F: A double inequality for the combination of Toader mean and the arithmetic mean in terms of the contraharmonic mean. Publ. Inst. Math. 99(113), 237-242 (2016)

41. Yang, Z-H, Chu, Y-M, Zhang, W: Monotonicity of the ratio for the complete elliptic integral and Stolarsky mean. J. Inequal. Appl. 2016, Article ID 176 (2016)

42. Vuorinen, M: Hypergeometric functions in geometric function theory. In: Special Functions and Differential Equations, Madras, 1997, pp. 119-126. Allied Publ., New Delhi (1998)

43. Barnard, RW, Pearce, K, Richards, KC: A monotonicity property involving ${ }_{3} F_{2}$ and comparisons of the classical approximations of elliptical arc length. SIAM J. Math. Anal. 32(2), 403-419 (2000)

44. Yang, Z-H, Chu, Y-M, Zhang, W: Accurate approximations for the complete elliptic integral of the second kind. J. Math Anal. Appl. 438(2), 875-888 (2016)

45. Yang, Z-H, Chu, Y-M, Zhang, X-H: Sharp Stolarsky mean bounds for the complete elliptic integral of the second kind J. Nonlinear Sci. Appl. 10(3), 929-936 (2017)

46. Yang, Z-H, Chu, Y-M, Tao, X-J: A double inequality for the trigamma function and its applications. Abstr. Appl. Anal. 2014, Article ID 702718 (2014)

47. Wendel, JG: Note on the gamma function. Am. Math. Mon. 55, 563-564 (1948)

48. Qi, F: Bounds for the ratio of two gamma functions. J. Inequal. Appl. 2010, Article ID 493058 (2010)

\section{Submit your manuscript to a SpringerOpen ${ }^{\ominus}$ journal and benefit from:}

- Convenient online submission

- Rigorous peer review

Immediate publication on acceptance

- Open access: articles freely available online

- High visibility within the field

- Retaining the copyright to your article 